\title{
Reddening and age for 13 southern Galactic open clusters determined from integrated spectra
}

\author{
A. V. Ahumada ${ }^{1}$, J. J. Clariá ${ }^{1}$, E. Bica ${ }^{2}$, C. M. Dutra ${ }^{3}$, and M. C. Torres ${ }^{1}$ \\ 1 Observatorio Astronómico, Universidad Nacional de Córdoba, Laprida 854, 5000 Córdoba, Argentina \\ e-mail: andrea@mail.oac.uncor.edu, mctorres@mail.oac.uncor.edu \\ 2 Instituto de Física, Universidade Federal do Rio Grande do Sul, Av. Bento Gonçalves, 9500, Caixa Postal 15051, \\ CEP 91501-970, Porto Alegre RS, Brazil \\ e-mail: bica@if.ufrgs.br \\ 3 Instituto Astronomico e Geofisico da USP, CP 3386, São Paulo 01060-970, Brazil \\ e-mail: dutra@andromeda.iagusp.usp.br
}

Received 6 July 2001 / Accepted 13 August 2001

\begin{abstract}
In this study we present flux-calibrated integrated spectra in the range 3800-6800 $\AA$ for 13 concentrated open clusters with Galactic longitudes between $219^{\circ}$ and $316^{\circ}$, nine of which have not been previously studied. Using the equivalent widths of the Balmer lines and comparing the cluster spectra with template spectra of Magellanic Clouds and Galactic star clusters with known parameters, we derive both foreground interstellar reddening values and age. For nine clusters these two parameters have been determined for the first time, while for the rest of the sample the results show good agreement with previous studies. The present analysis indicates four very young (Hogg 11, NGC 5606, vdB-RN 80 and Pismis 17), seven moderately young (ESO 429-SC13, Hogg 3, Hogg 12, Haffner 7, BH 87, NGC 2368 and Bochum 12) and two intermediate-age (Berkeley 75 and NGC 2635) open clusters. The derived foreground interstellar reddening values are in the range $0.00 \leq E(B-V) \leq 0.38$. The age and reddening distributions of the present sample of relatively faint open clusters match those of open clusters with known parameters in a $90^{\circ}$ sector centered at $l=270^{\circ}$.
\end{abstract}

Key words. methods: observational - techniques: spectroscopic - open clusters and associations: general

\section{Introduction}

Open clusters are fundamental landmarks to probe the Galaxy properties. They are among the very few Galactic objects for which meaningful distances can be derived over a large range, which makes them an essential tool to constrain Galactic evolution theories. They also provide more accurate ages than do other disk objects. Therefore, the study of the Galactic open cluster system is very useful for many purposes concerning our Galaxy's structure (Janes \& Adler 1982) and evolution (Friel \& Janes 1993; Piatti et al. 1995).

The open cluster catalogue by Lyngå (1987) contains 1151 entries. However, very little is known for many of them, except their positions and estimates of their angular

Send offprint requests to: J. J. Clariá,

e-mail: claria@mail.oac.uncor.edu

* Based on observations made at Complejo Astronómico El Leoncito, which is operated under agreement between the Consejo Nacional de Investigaciones Científicas y Técnicas de la República Argentina and the National Universities of La Plata, Córdoba and San Juan, Argentina. sizes. Despite the fact that reddening, age and metallicity determinations for Galactic open clusters have been based mostly on colour-magnitude diagrams (CMDs) and/or photometric studies of individual giants (see, e.g., Janes et al. 1988; Meynet et al. 1993; Clariá et al. 1999), integrated spectroscopy (e.g., Santos \& Bica 1993; Piatti et al. 1999) can also be an effective source of valuable independent information about these parameters in compact objects.

The Milky Way region in a $90^{\circ}$ sector centered at $l=270^{\circ}$ is especially rich in open clusters, many of which have not yet been the object of previous research. As part of a systematic spectroscopic survey of concentrated open clusters, the current study seeks to derive interstellar reddening values and ages for a sample of open clusters located within the above mentioned sector. In the next section we present the cluster sample and the spectroscopic observations. The methods employed to derive age and reddening values, as well as the measurements of equivalent widths for Balmer absorption features are described in Sect. 3. A discussion of individual clusters is provided in Sect. 4. A comparison with other clusters located in 
similar directions is given in Sect. 5, and the final conclusions are presented in Sect. 6.

\section{Cluster sample and spectroscopic observations}

Galactic open clusters have in general comparatively large angular sizes and low surface brightness. Nevertheless, many of them appear to be concentrated objects which lend themselves readily to integrated spectroscopic observations. Of the 306 open clusters with angular diameters $D \leq 4^{\prime}$ in the Lyngå (1987) catalogue, only $48(15 \%)$ have information about reddening and age. In this study we have selected 13 relatively populous, small angular diameter $\left(D \leq 4^{\prime}\right)$ open clusters with Galactic longitudes ranging between $219^{\circ}$ and $316^{\circ}$, nine of which have not been previously studied. The observed star cluster sample is given in Table 1, where cluster designations in different catalogues are provided. Galactic and equatorial coordinates for the cluster sample are listed in Table 1, together with the angular sizes taken from Lyngå (1987), Lauberts (1982) or estimated by ourselves on Digitized Sky Survey images. The objects studied here are part of a systematic spectroscopic survey of open and globular clusters (or candidate globular) which is being undertaken at Complejo Astronómico El Leoncito (CASLEO) in San Juan (Argentina). The first results of this survey dealt with 3 globular cluster candidates, two of which turned out to be planetary nebulae, the remaining one being very probably a supernova remnant (Bica et al. 1995). Fundamental parameters for many Galactic open and globular clusters have been derived during the last three years from integrated spectroscopic observations undertaken at CASLEO (Bica et al. 1998; Piatti et al. 1998a, 1998b, 1999, 2000a, 2000b; Ahumada et al. 2000).

The observations analysed in this study were carried out with the CASLEO $2.15 \mathrm{~m}$ telescope during six nights in February 2000 and two nights in May 2001. We employed a charge-coupled device (CCD) camera attached to the REOSC spectrograph during the two runs. The detector was a Tektronics chip of $1024 \times 1024$ pixels of size $24 \times 24 \mu^{2}$; one pixel corresponds to $0.94^{\prime \prime}$ on the sky. We have used the coordinates from Table 1 only to point to the object. The slit was then set in the East-West direction and the observations were performed by scanning the slit across the objects in the North-South direction in order to get a proper sampling of cluster stars. We used a grating of 300 grooves $\mathrm{mm}^{-1}$, producing an average dispersion, in the observed region, of $\approx 140 \AA / \mathrm{mm}(3.46 \AA /$ pix $)$. The spectral coverage was $\approx 3800-6800 \AA$. The long slit, corresponding to $4.7^{\prime}$ on the sky, allowed us to sample regions of background sky. The seeing during the nights was typically $2.5^{\prime \prime}$. The slit width was $4.2^{\prime \prime}$, providing a resolution [full width at half-maximum (FWHM)] of $\approx 17 \AA$, as deduced from the comparison lamp lines. The standard stars LTT 2415, LTT 3864, CD-32 9927, LTT 6248, EG 274 and LTT 7987 (Stone \& Baldwin 1983) were observed for flux calibrations. Bias, darks, dome and twilight sky and tungsten lamp flats were taken and employed in the reductions.

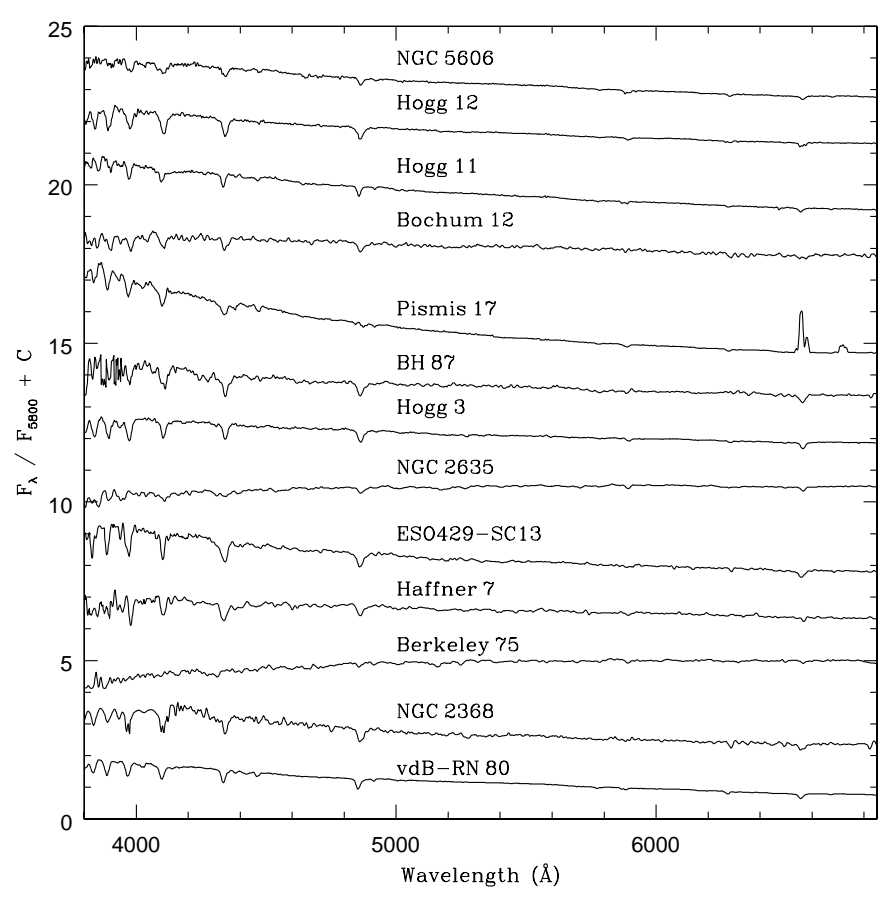

Fig. 1. Observed integrated spectra of the sample clusters. Spectra are in relative $F_{\lambda}$ units normalized at $5800 \AA$. Constants have been added to the spectra, except for the bottom one.

A series of exposure times of $15 \mathrm{~min}$ each were employed for the objects giving a total of $60 \mathrm{~min}$ for each observed cluster. Reductions were carried out with the IRAF software package, following standard procedures at the Observatorio Astronómico de la Universidad Nacional de Córdoba (Argentina). The spectra were extracted along the slit according to the cluster size and available flux. We subtracted the bias and used flat-field frames - previously combined - to correct the frames for high and low spatial frequency variations. Background sky subtractions were then performed using pixel rows from the same frame after removing cosmic rays from the background sky regions, taking the precaution that no significant background sky residuals were present on the resulting spectra. Wavelength calibrations were carried out with a $\mathrm{Cu}-\mathrm{He}-\mathrm{Ar}$ lamp with exposures following that of the object or standard star. The rms errors involved in these calibrations are typically $0.70 \AA$ or 0.20 pixel. Atmospheric extinction corrections according to the site coefficients given by Minniti et al. (1989) and flux calibrations were then applied.

Flux-calibrated integrated spectra of the observed clusters are shown in Fig. 1. Spectra are in relative $F_{\lambda}$ units normalized at $\lambda \approx 5800 \AA$ and have been shifted by different constants (except for the bottom one) for comparison purposes. The signal-to-noise $(\mathrm{S} / \mathrm{N})$ ratios of the spectra, measured at the same continuum region, have a typical value of 30 , which allows us to distinguish absorption features differing around $10 \%$ of the continuum intensity. Note that while some amount of field star contamination may be expected in the observed spectra, only bright field stars could affect the spectra significantly. A bright 
Table 1. Cluster sample.

\begin{tabular}{|c|c|c|c|c|c|}
\hline Cluster & $\begin{array}{c}l \\
\left({ }^{\circ}\right)\end{array}$ & $\begin{array}{c}b \\
\left({ }^{\circ}\right)\end{array}$ & $\begin{array}{c}\alpha_{2000} \\
(\mathrm{~h} \mathrm{~m} \mathrm{~s})\end{array}$ & $\begin{array}{l}\delta_{2000} \\
\left(\begin{array}{lll}0 & \prime \prime\end{array}\right)\end{array}$ & $\begin{array}{l}D \\
\left({ }^{\prime}\right)\end{array}$ \\
\hline vdB-RN 80 & 219.56 & -8.44 & $6: 30: 46$ & $-09: 40: 09$ & 4 \\
\hline NGC 2368, Cr 138 & 225.89 & 2.21 & $7: 20: 58$ & $-10: 22: 41$ & 3 \\
\hline Berkeley 75, ESO 490-SC50 & 234.56 & -10.71 & $6: 49: 16$ & $-23: 57: 29$ & 4 \\
\hline Haffner 7, BH 1, ESO 428-SC24 & 242.99 & -6.42 & $7: 23: 10$ & $-29: 29: 51$ & 4 \\
\hline ESO $429-\mathrm{SC} 13$ & 245.95 & -3.63 & $7: 41: 03$ & $-30: 44: 03$ & 3 \\
\hline NGC 2635, BH 39, Mel 89, Cr 190, ESO 371-SC1 & 255.98 & 4.18 & $8: 38: 28$ & $-34: 45: 35$ & 3.5 \\
\hline Hogg 3, ESO 167-SC12 & 279.86 & 0.09 & 9:57:51 & $-54: 39: 21$ & 2 \\
\hline $\mathrm{BH} 87$ & 281.03 & -0.03 & 10:03:56 & $-55: 26: 34$ & 3 \\
\hline Pismis 17 & 289.81 & -0.00 & 11:01:04 & $-59: 49: 08$ & 3 \\
\hline Bochum 12 & 290.19 & -1.93 & $10: 57: 23$ & $-61: 44: 03$ & 2.5 \\
\hline Hogg 11, ESO 129-SC6 & 291.23 & -0.01 & $11: 11: 32$ & $-60: 22: 18$ & 2.5 \\
\hline Hogg 12, ESO 129-SC11 & 291.46 & -0.33 & $11: 12: 20$ & $-60: 45: 19$ & 2 \\
\hline NGC 5606, BH 158, Cr 281, ESO 134-SC3 & 315.19 & 0.61 & $14: 27: 47$ & $-59: 38: 25$ & 3.5 \\
\hline
\end{tabular}

star is superimposed on the cluster NGC 2368 (possibly an atypical luminous member; see Sect. 4.2), so that in this case different spatial extractions were considered.

\section{Reddening and age determinations}

Integrated spectra of open clusters of small angular diameter allow one to determine their basic properties, such as reddening, age and metallicity. Bica \& Alloin (1986a, 1987, herefater BA86a and BA87, respectively) have studied integrated spectra in the blue-visible and near-IR ranges of Galactic open and globular clusters, as well as Magellanic Cloud clusters. They examined the behaviour of metallic and Balmer line equivalent widths, as well as the continuum energy distribution in the spectral range 3700 $10000 \AA$ A. They also generated a library of template cluster spectra with well-known properties. Spectral libraries of template spectra grouped according to their evolutionary stages have also been published by Bica (1988), Bica et al. (1990), Bonatto et al. (1995) and Santos et al. (1995).

We determined the fundamental parameters of the observed open clusters from their integrated spectra using the SPEED spectral analysis software (Schmidt 1988). A direct age estimate was first obtained from equivalent widths $(\mathrm{EWs})$ of the Balmer lines in absorption in each spectrum by interpolating these values in the age calibration of Bica \& Alloin (1986b, hereafter BA86b). The ages derived by this procedure are reddening independent. We then selected an appropriate set of template spectra according to the ages provided by the Balmer lines and varied reddening and template to obtain the best match of continuum and lines of the observed spectra to that of the template that most resembles it. The reddening corrections were performed using the interstellar absorption law derived by Seaton (1979) and adopting the factor 3.0 for the ratio of total-to-selective extinction, i.e., $A_{v}=3.0 E(B-V)$. We note that the uncertainty in the adopted reddening represents the lowest reddening variation necessary to distinguish the cluster spectrum from that of the corresponding template. As shown in Table 3, the uncertainties in the $E(B-V)$ determinations range from 0.02 to 0.05 magnitudes. If the age assignment were off by one template class, the above uncertainties could be only slightly larger.

In order to measure EWs of the Balmer lines, we have taken into account both the spectral windows and flux points, as defined by BA86a, BA86b and BA87. The errors affecting the derived EWs were estimated from different measurements of the Balmer lines using high and low continuum tracings in order to take into account the spectral noise. The resulting uncertainties in the EWs are smaller than those associated with the intrinsic dispersions in Table 2 of BA86b. The results of the measurements are shown in Table 2, where the EWs are given in Angström units $(\AA)$.

The following template groups from Bica (1988) and Santos et al. (1995) were found to be useful in this study: YA.LMC (3-6 Myr), YC.LMC (12-40 Myr), YDE.LMC (35-65 Myr), Y3A (100 Myr), Y3B (100 Myr, but with an enhanced population of massive asymptotic giant branch stars with respect to Y3A), I1 (1 Gyr) and I2 (2 Gyr). Interestingly, the template sequence depends primarily on age, the metallicity effects being negligible in the spectral and age ranges observed in the current study, except for the intermediate-age cluster.

\section{Discussion on individual clusters}

The open clusters vdB-RN 80, NGC 2368, Berkeley 75, Haffner 7, ESO 429-SC13, NGC 2635, Hogg 3, BH 87 and Hogg 12 have not been the target of existing research, so their foreground reddening values and ages have been determined here for the first time. There exist previous results only about Pismis 17, Bochum 12, Hogg 11 and NGC 5606, which can be compared with the present ones. Data from previous studies were obtained from the WEBDA (http://obswww. unige.ch/webda/) open cluster database of Mermilliod $(1988,1992)$. In this section we briefly discuss the results obtained for each cluster. 
Table 2. Measurements of equivalent widths of four Balmer lines.

\begin{tabular}{lcccc}
\hline & $\mathrm{H}_{\delta}$ & $\mathrm{H}_{\gamma}$ & $\mathrm{H}_{\beta}$ & $\mathrm{H}_{\alpha}$ \\
Windows & $(4082-4124) \AA$ & $(4318-4364) \AA$ & $(4846-4884) \AA$ & $(6540-6586) \AA$ \\
Cluster & & & & \\
\hline vdB-RN 80 & $6.46 \pm 0.07$ & $5.62 \pm 0.09$ & $5.6 \pm 0.1$ & $5.0 \pm 0.1$ \\
NGC 2368 & $5.6 \pm 0.3$ & $8.8 \pm 0.1$ & $7.6 \pm 0.1$ & $6.97 \pm 0.07$ \\
Berkeley 75 & $5.8 \pm 0.3$ & $5.0 \pm 0.1$ & - & - \\
Haffner 7 & $10.9 \pm 0.1$ & $9.28 \pm 0.04$ & $6.8 \pm 0.1$ & $3.65 \pm 0.06$ \\
ESO 429-SC13 & $8.4 \pm 0.3$ & $11.1 \pm 0.3$ & $9.4 \pm 0.4$ & $7.5 \pm 0.2$ \\
NGC 2635 & $8.0 \pm 0.1$ & $8.6 \pm 0.2$ & $5.1 \pm 0.1$ & $3.13 \pm 0.03$ \\
Hogg 3 & $7.8 \pm 0.1$ & $7.38 \pm 0.05$ & $7.1 \pm 0.1$ & $6.03 \pm 0.07$ \\
BH 87 & $10.4 \pm 0.1$ & $14.2 \pm 0.3$ & $8.7 \pm 0.2$ & $7.41 \pm 0.06$ \\
Pismis 17 & $5.2 \pm 0.1$ & $3.70 \pm 0.05$ & $1.82 \pm 0.06$ & $-28.7 \pm 0.08$ \\
Bochum 12 & $10.0 \pm 0.2$ & $7.09 \pm 0.05$ & $8.5 \pm 0.2$ & $4.1 \pm 0.1$ \\
Hogg 11 & $5.0 \pm 0.1$ & $4.38 \pm 0.05$ & $4.38 \pm 0.09$ & $4.00 \pm 0.05$ \\
Hogg 12 & $9.6 \pm 0.1$ & $8.37 \pm 0.07$ & $7.0 \pm 0.1$ & $4.75 \pm 0.05$ \\
NGC 5606 & $5.22 \pm 0.09$ & $3.71 \pm 0.08$ & $5.22 \pm 0.09$ & $4.32 \pm 0.06$ \\
\hline
\end{tabular}

Table 3. Reddening and age determinations.

\begin{tabular}{lcccc}
\hline Cluster & $E(B-V)$ & $\begin{array}{c}\text { Balmer Age } \\
(\mathrm{Myr})\end{array}$ & $\begin{array}{c}\text { Template Age } \\
(\mathrm{Myr})\end{array}$ & $\begin{array}{c}\text { Adopted age } \\
(\mathrm{Myr})\end{array}$ \\
\hline vdB-RN 80 & $0.38 \pm 0.04$ & $30 \pm 10$ & $3-6$ & $4.5 \pm 1.5$ \\
NGC 2368 & $0.12 \pm 0.03$ & $60 \pm 30$ & $35-65$ & $50 \pm 10$ \\
Berkeley 75 & $0.05 \pm 0.02$ & $4000 \pm 1000$ & 2000 & $3000 \pm 1000$ \\
Haffner 7 & $0.10 \pm 0.05$ & $120 \pm 60$ & 100 & $100 \pm 10$ \\
ESO 429-SC13 & $0.00 \pm 0.03$ & $125 \pm 100$ & 100 & $100 \pm 50$ \\
NGC 2635 & $0.05 \pm 0.03$ & $2000 \pm 1000$ & 1000 & $1500 \pm 500$ \\
Hogg 3 & $0.15 \pm 0.02$ & $50 \pm 10$ & 100 & $75 \pm 25$ \\
BH 87 & $0.10 \pm 0.02$ & $200 \pm 100$ & 100 & $150 \pm 50$ \\
Pismis 17 & $0.19 \pm 0.02$ & $15 \pm 5$ & $3-6$ & $4.5 \pm 1.5$ \\
Bochum 12 & $0.30 \pm 0.02$ & $60 \pm 10$ & $12-40$ & $45 \pm 15$ \\
Hogg 11 & $0.24 \pm 0.04$ & $15 \pm 5$ & $3-6$ & $8 \pm 5$ \\
Hogg 12 & $0.04 \pm 0.02$ & $70 \pm 20$ & 100 & $85 \pm 15$ \\
NGC 5606 & $0.31 \pm 0.03$ & $35 \pm 20$ & $3-6$ & $4.5 \pm 1.5$ \\
\hline
\end{tabular}

\section{1. vdB-RN 80 Cluster}

A catalogue of 158 reflection nebulae, associated with $\mathrm{BD}$ or $\mathrm{CD}$ stars north of $\delta=-33^{\circ}$, was published by van den Bergh (1966). Object number 80 in this catalogue refers to a reflection nebula in Monoceros which harbours a star cluster including the stars BD- $9^{\circ} 1947$ and BD- $9^{\circ} 1948$. The integrated spectrum of vdB-RN 80 , corrected for $E(B-V)=0.38$, is compared to that of the YA.LMC template $(3-6 \mathrm{Myr})$ in Fig. 2. Although the Balmer lines indicate a somewhat larger age (Table 3), we adopted the template method age for this cluster because Balmer lines lose sensitivity for very young objects (BA86a). This is the most reddened cluster in the sample, which can be explained by its association to dust as denoted by the occurrence of the reflection nebula.

\subsection{NGC 2368}

This cluster, also known as Cr 138 (Collinder 1931), presents the comparatively bright variable star V632 Mon (Kholopov et al. 1998) close to its centre, which dominates the integrated spectrum. Therefore, we analysed two spatial extractions: that of the cluster spectrum (without the above bright star), corresponding to $90^{\prime \prime}$ along the slit (Fig. 3), and that of the dominating star spectrum, corresponding to $30^{\prime \prime}$ along the slit (Fig. 4). The latter, classified as an M7III-M9 star (Kholopov et al. 1998), presents strong $\mathrm{TiO}$ bands comparable to those seen in the star HD 142143, classified as an M6-7S III star in the Spectroscopic Atlas by Silva \& Cornell (1992). V632 Mon could be an asymptotic giant branch (AGB) star with a relatively massive star as progenitor. The possibility of the bright late-type giant being a cluster member is not 


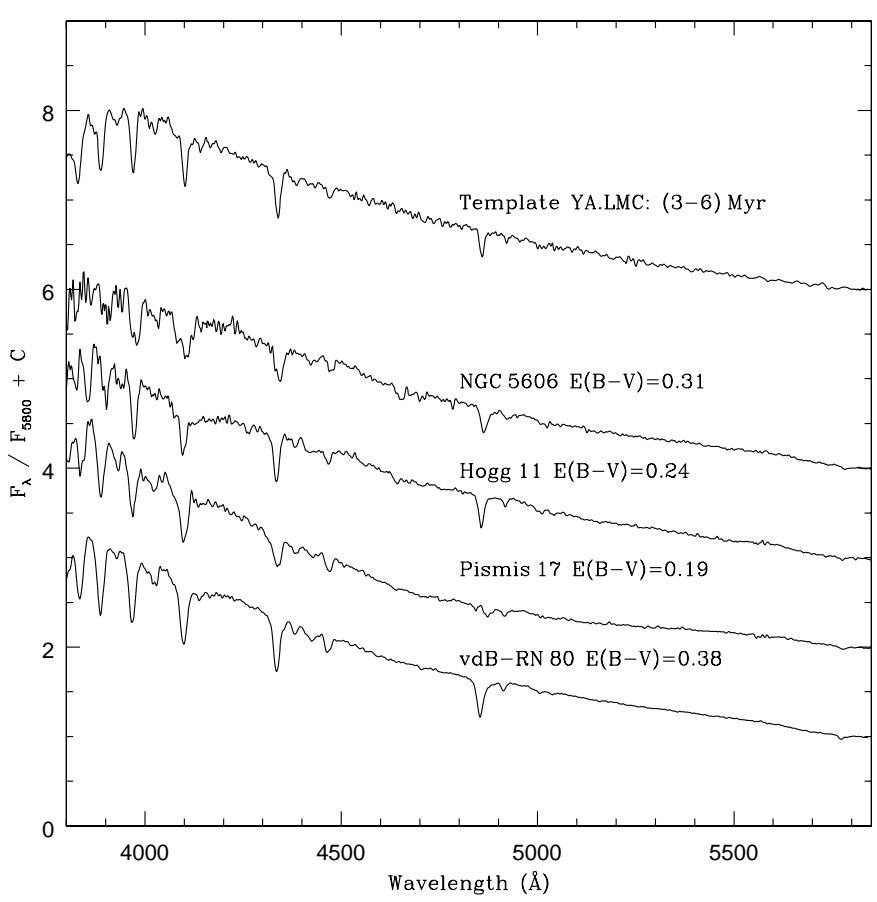

Fig. 2. Reddening-corrected integrated spectra of vdB-RN 80, Pismis 17, Hogg 11 and NGC 5606, and the YA.LMC template which best matches these spectra (top). Units as in Fig. 1.

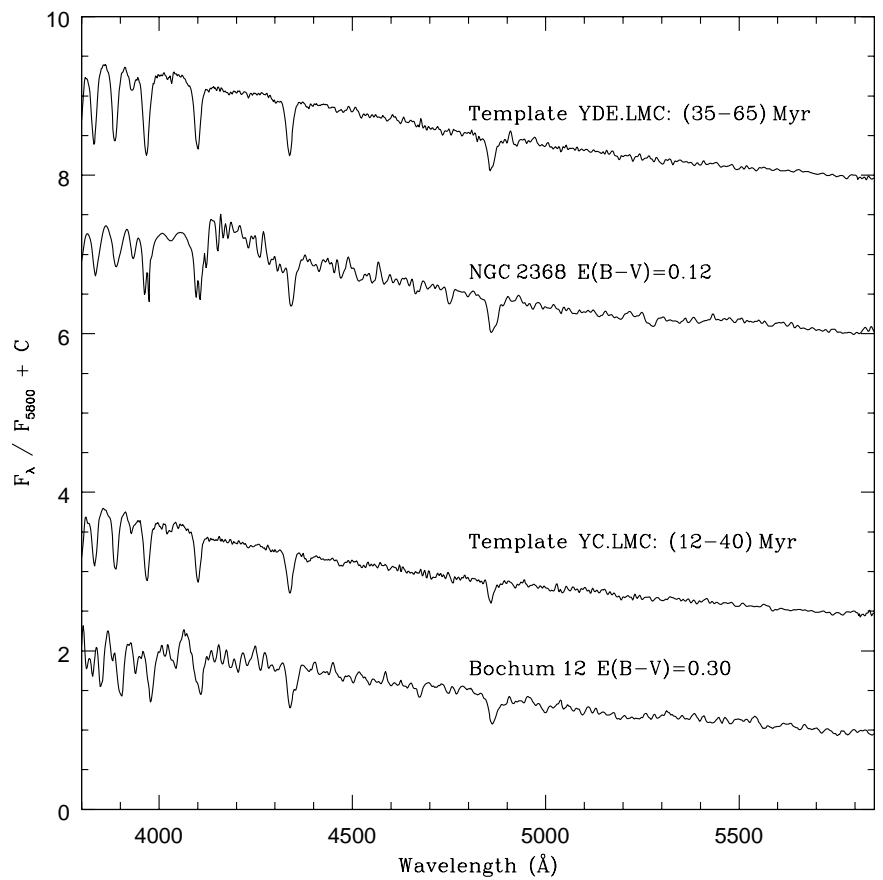

Fig. 3. Reddening-corrected integrated spectra of NGC 2368 compared with the YDE.LMC template (top), and Bochum 12 compared with the YC.LMC template (bottom). Units as in Fig. 1.

excluded. An example of a $\approx 100$ Myr cluster with luminous M-type AGB stars is NGC 1866 in the Large Magellanic Cloud (Frogel et al. 1990). The EWs of the Balmer lines in the cluster spectrum indicate an age near

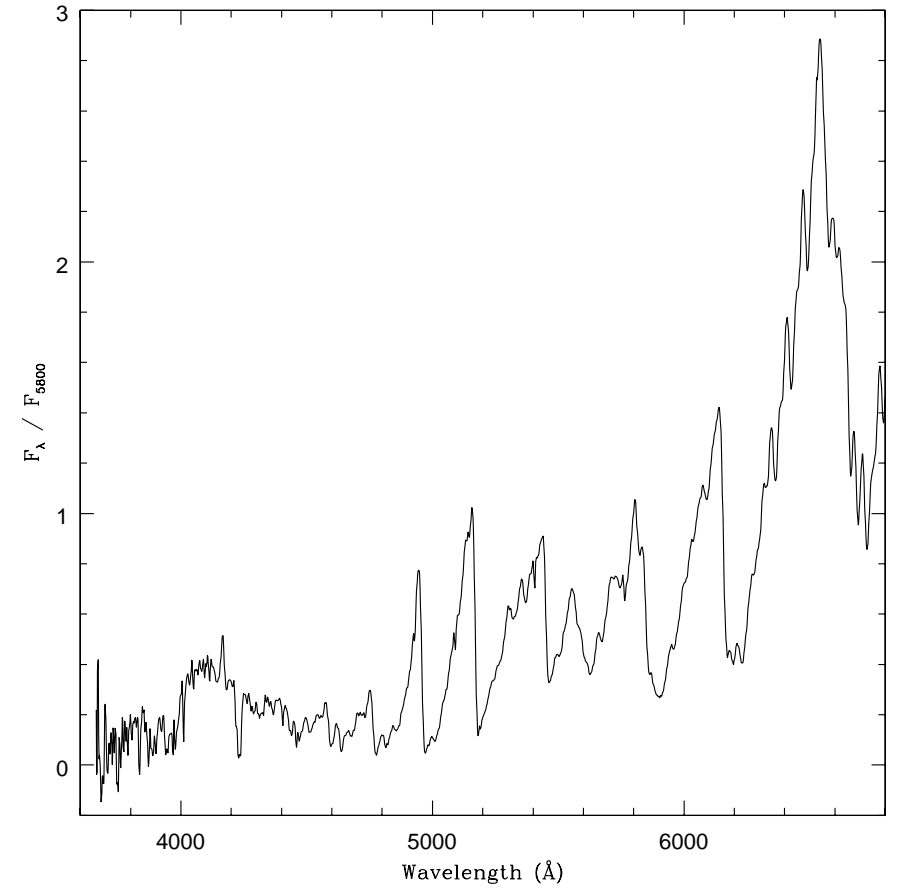

Fig. 4. Spectrum of the dominating star of the cluster NGC 2368. Units as in Fig. 1.

$60 \mathrm{Myr}$, while the best match is found for the YDE.LMC template of similar age, using $E(B-V)=0.12$ (Fig. 3).

\subsection{Berkeley 75}

Despite the $\mathrm{S} / \mathrm{N}$ ratio being rather low, the integrated spectrum of Berkeley 75 or ESO 490-SC50 (Lauberts 1982) shows typical features of intermediate-age clusters (Fig. 1). Note the metal lines, in particular MgI $5175 \AA$ and FeI $5276 \AA$, which indicate that the cluster is older than the Y4 template of 500 Myr. As far as the younger clusters are concerned, they have their blue-visible region dominated by the upper main sequence. Unlike the young clusters, the intermediate-age clusters (IACs) have their optical integrated light dominated by giants, but the turnoff stars may be important contributors as denoted by the Balmer lines, especially for ages near 1 Gyr. We obtained the best comparison with the intermediate-age I2 template (2 Gyr), correcting the observed spectrum for a small amount of reddening: $E(B-V)=0.05$ (Fig. 5).

\subsection{Haffner 7}

This object, also referred to as BH1 (van den Bergh \& Hagen 1975) or ESO 428-SC24 (Lauberts 1982), was first identified as an open cluster by Haffner (1957). Figure 6 shows the observed integrated spectrum, corrected for $E(B-V)=0.10$, compared to the Y3A template (100 Myr). Since the Balmer-line method also furnishes an age closer to that of the above template (Table 3), we have adopted the latter as the best solution. 


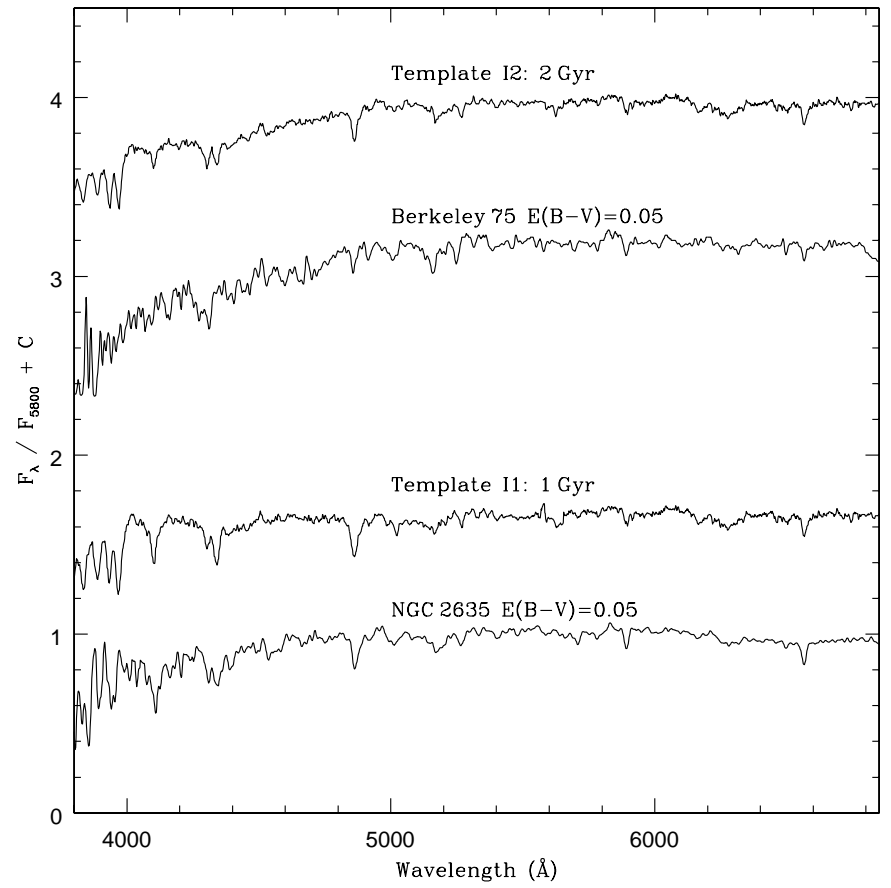

Fig. 5. Reddening-corrected integrated spectra of Berkeley 75 compared with the I2 template (top), and NGC 2635 compared with the I1 template (bottom). Units as in Fig. 1.

\subsection{ESO429-SC 13}

This neglected cluster in Puppis was first identified by Holmberg et al. (1978) in the VI list of the ESO/Uppsala survey. All survey lists were later merged into a single catalogue (Lauberts 1982). The spectral features of this cluster are quite similar to those of Hogg 3. A good match is found with the Y3B template (100 Myr), using $E(B-V)=0.00$ (Fig. 6).

\subsection{NGC 2635}

This cluster is also known as BH39 (van den Bergh \& Hagen 1975), Melotte 89 (Melotte 1915), Cr 190 (Collinder 1931) or ESO 371-SC1 (Lauberts 1982). Based on $U B V$ photoelectric data of scarcely six comparatively bright stars in the cluster field, Vogt \& Moffat (1972) suggested that this is probably a random gathering of field stars in Pyxis rather than an open cluster. Their $(U-B)$ versus $(B-V)$ diagram reveals that there are no early-type stars among those observed. It is important to note, however, that if we are dealing with an old or intermediate-age cluster, their brightest members should be late-type stars, such as those observed by Vogt \& Moffat (1972). Likewise for Berkeley 75, the integrated spectrum of NGC 2635 shows typical features of intermediate-age clusters. In Fig. 5 we show the reddening-corrected spectrum for $E(B-V)=0.05$ and the I1 template (1 Gyr) which best matches the spectrum. Due to the fact that the Balmerline method suggests $\approx 2 \mathrm{Gyr}$, we have adopted an age of 1.5 Gyr for NGC 2635.

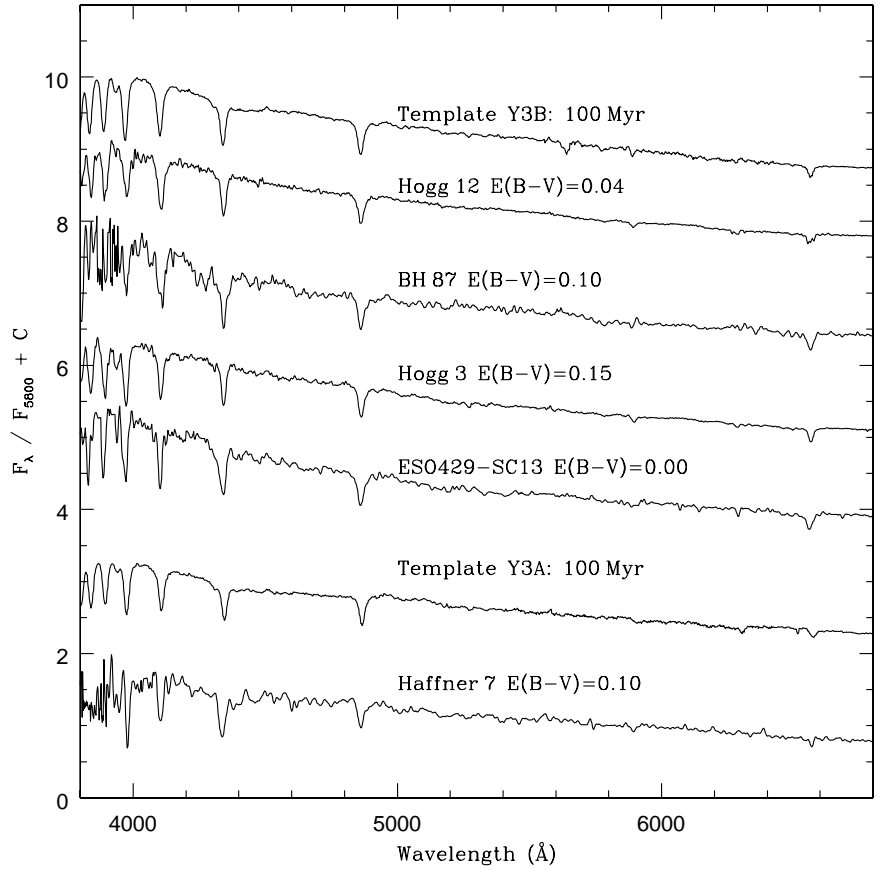

Fig. 6. Observed integrated spectrum of ESO 429-SC13 and the reddening-corrected integrated spectra of $\operatorname{Hogg} 3, \mathrm{BH} 87$ and Hogg 12 compared with the Y3B template (top), and Haffner 7 compared with the Y3A template (bottom). Units as in Fig. 1.

\section{7. $\operatorname{Hogg} 3$}

As far as we are aware, no previous data exist for this Vela object firstly reported as an open cluster by Hogg (1965a, 1965b). Figure 6 shows the integrated cluster spectrum, corrected for $E(B-V)=0.15$, compared to the Y3B template (100 Myr). A general resemblance between these two spectra is clearly apparent. Although the age from Balmer lines is slightly smaller (Table 3), Hogg 3 or ESO 167-SC12 (Lauberts 1982) is found to be a moderately young open cluster.

\section{8. $\mathrm{BH} 87$}

This object was recognized as an open cluster by van den Bergh \& Hagen (1975). Its integrated spectral properties are comparable to those of Hogg 3 and ESO 429SC13 (Fig. 6). The solution for $\mathrm{BH} 87$ yields an age of about $100 \mathrm{Myr}$ and $E(B-V)=0.10$, although Balmer lines suggest an age slightly larger (Table 3 ).

\subsection{Pismis 17}

Pismis 17 is embedded in the small bright nebula NGC 3503 (=ESO128-EN28, Lauberts 1982). This nebula, besides emission, has a reflection component, and it is also kown as vdBH-RN46 in the southern reflection nebulae catalogue by van den Bergh \& Herbst (1975). NGC 3503, in turn, is embedded (or in the background) of Gum 34b (Gum 1955), the latter itself being part of the large 
complex RCW 54 (Rodgers et al. 1960) in Carina. Pismis 17 was first recognized as a compact open cluster by Pismis (1959). Moffat \& Vogt (1975) obtained $U B V$ photoelectric data for 9 stars in the cluster field, two of them being very probably field stars, according to their positions in the CMD and colour-colour diagram. They derived a foreground reddening $E(B-V)=0.51 \pm 0.07$ and a cluster distance of $4.2 \mathrm{kpc}$ from the Sun, the earliest photometric spectral type being b0.5. Although Pismis 17 is nearly $1.5^{\circ}$ northwest of the Carina OB2 association, it is very probably not physically connected with this association since the latter is located at a distance of $3.1 \mathrm{kpc}$ from the Sun (García et al. 1988; García 1994).

Figure 2 shows the integrated cluster spectrum, corrected for $E(B-V)=0.19$, compared to the YA.LMC template (3-6 Myr). A general resemblance between these two spectra is seen at wavelengths shorter than $\approx 4700 \AA$. However, clear differences do appear when the first two Balmer lines are compared in both spectra. In fact, $\mathrm{H}_{\alpha}$ is in emission (Fig. 1), while $\mathrm{H}_{\beta}$ is hardly visible in the cluster spectrum due to the gas emission partially filling the stellar absorption. Although the age derived from the $\mathrm{H}_{\gamma}$ and $\mathrm{H}_{\delta}$ Balmer lines is slightly larger than that provided for the best template match, we have adopted the latter for the same reasons as for vdB-RN 80 (Sect. 4.1). Note that the spectrum of Pismis 17 has nebular lines such as [SII] $6717,6730 \AA$ (Fig. 1), while vdB-RN 80 has none, both being embedded clusters of similar age. This indicates that vdB-RN 80 is a cluster lacking massive stars, and in turn an HII region. As Pismis 17 is associated to nebulosity, it is highly probable that the difference $\Delta E(B-V)=0.32$ between the reddening derived by Moffat \& Vogt (1975) and the one here obtained, be entirely due to internal reddening. Indeed, if there exists internal dust associated to the cluster, then the spectroscopic $E(B-V)$ value should be lower than the photometric one, since the less reddened stars of a given spectral type should contribute to the integrated light with larger fluxes in comparison with the most reddened stars of the same spectral type. Note also that templates are not corrected for interstellar reddening (Sect. 4.13).

\subsection{Bochum 12}

This is a compact group of stars around the OB star SLS 2054 from the Catalogue of Luminous Stars in the Southern Milky Way (SLS) by Stephenson \& Sanduleak (1971). Moffat \& Vogt (1975) reported UBV photoelectric photometry for 12 comparatively bright stars in the cluster field, 7 of which seem to form a cluster. They derived a foreground reddening $E(B-V)=0.24 \pm 0.03$ and a cluster distance from the Sun of $2.22 \mathrm{kpc}$, the earliest photometric spectral type being b5. Figure 3 shows the cluster integrated spectrum, corrected for $E(B-V)=0.30 \pm 0.05$, compared to the YC.LMC template $(\approx 25 \mathrm{Myr})$ which best matches the cluster spectrum. The template age is compatible with that provided by the Balmer lines (Table 3).

\subsection{1. $\operatorname{Hogg} 11$}

Hogg 11, also known as ESO 129-SC6 (Lauberts 1982), is a small group of stars south of NGC 3572 in Carina, first recognized as an open cluster by Hogg (1965a, 1965b). This compact cluster, together with NGC 3590, Hogg 10, Cr 240, NGC 3572, Trumpler 18 and Hogg 12, forms an extensive and almost continuous grouping of moderately bright stars lying in a region where the Carina spiral feature is seen tangentially (see, e.g., Clariá 1976). Moffat \& Vogt (1975) observed photoelectrically in the $U B V$ system only 6 comparatively bright stars in the cluster field, of which only the three brightest ones might be cluster members. They derived $E(B-V)=0.32 \pm 0.01$ and a cluster distance of $2.3 \mathrm{kpc}$, the earliest photometric spectral type being b1. The two Balmer lines with the highest detection level $\mathrm{EW} / \Delta \lambda$ yield an age of about $15 \mathrm{Myr}$, while the best match is found with the YA.LMC template (3-6 Myr), using $E(B-V)=0.24$ (Fig. 2). We then adopted the weighted mean value of $8 \pm 5$ Myr for Hogg 11, which is quite consistent with the earliest photometric spectral type suggested by Moffat \& Vogt (1975). The larger reddening derived from the photometric data of Moffat \& Vogt (1975) could be indicating an internal cluster reddening of $\approx 0.08$ mag.

\subsection{2. $\operatorname{Hogg} 12$}

This Carina cluster, also known as ESO 129-SC11 (Lauberts 1982), was first recognized as an open cluster by Hogg (1965a, 1965b). Nonetheless, based on $U B V$ photoelectric photometry of 11 stars in the cluster field, Moffat \& Vogt (1975) considered this sparse group of stars as a probable random fluctuation of the field star density. The solution for Hogg 12 yields an age of about $100 \mathrm{Myr}$ and $E(B-V)=0.04$ by template match (Fig. 6). The age derived from the Balmer lines is only slightly smaller (Table 3). The spectral features of Hogg 12 are quite similar to those of the more reddened cluster Hogg 3 (Sect. 4.7).

\subsection{NGC 5606}

NGC 5606, also known as BH 158 (van den Bergh \& Hagen 1975) or Cr 281 (Collinder 1931), is a small size grouping of early-type stars situated in a low absorption zone in the Centaurus region (Feinstein 1994). Based on $U B V$ photoelectric photometry of 15 stars, Moffat \& Vogt (1973) showed the interstellar reddening in front of this cluster to be variable, the mean value being $\langle E(B-V)\rangle=0.49$. On the other hand, using UBVRI photoelectric and deep CCD photometry in the cluster field, Vázquez \& Feinstein (1991) and Vázquez et al. (1994), respectively, derived an age of 6.5 Myr and a mean reddening $E(B-V)=0.51$. The measured full width of the $(U-B)$ vs. $(B-V)$ diagram observed by Vázquez \& Feinstein (1991) is $\Delta E(B-V)=$ $E(B-V)_{\max }-E(B-V)_{\min }=0.27$, i.e., much larger than 
A. V. Ahumada et al.: Fundamental parameters of 13 Galactic open clusters

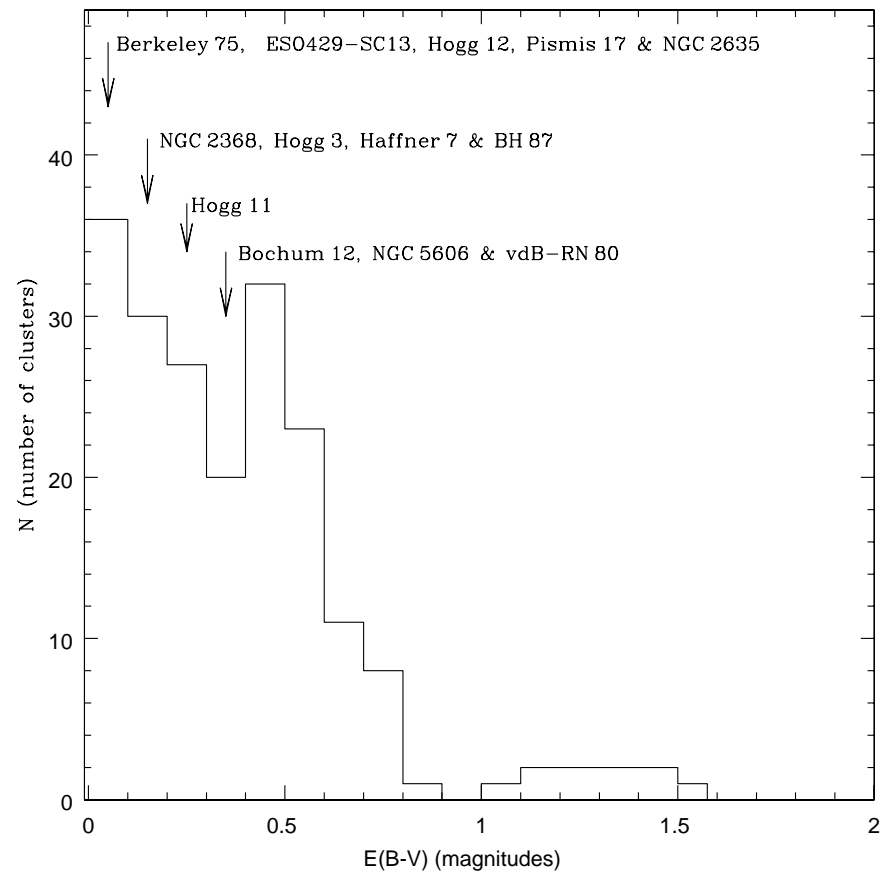

Fig. 7. Reddening histogram in the $90^{\circ}$ Galactic sector centered at $l=270^{\circ}$.

the lower limit of 0.11 mag estimated by Burki (1975) for clusters with differential reddening.

We have simultaneously determined the age and reddenig for NGC5606 using the template YA.LMC (3-6 Myr), the best template match yielding $E(B-V)=$ 0.31 (Fig. 2). Although the Balmer lines with the highest detection level indicate a somewhat larger age (Table 3), they do lose sensitivity for very young objects (BA86a). Accordingly, we then adopted the template method age for NGC 5606. The age derived here is in very good agreement with that obtained by Vázquez \& Feinstein (1991) and Vázquez et al. (1994). As expected, however, the spectroscopic reddening derived in this study is compatible with the smallest individual $E(B-V)$ values derived by Vázquez \& Feinstein (1991), since the adopted template integrated spectra are not corrected for internal reddening, which is increasingly important among younger ages.

\section{Comparison with other open clusters}

Ahumada et al. (2000) carried out a similar analysis, by deriving ages and metallicities for a relatively faint new sample of clusters and comparing them to all available literature values for clusters younger than $600 \mathrm{Myr}$ in $90^{\circ}$ sectors centered at $l=270^{\circ}$ and $l=0^{\circ}$, respectively. They found that two clusters (Hogg 15 and Pismis 21) appear to be among the most reddened optical open clusters in sectors centered at $l=270^{\circ}$ and $l=0^{\circ}$, respectively. They also found that, unless major star forming events had occurred in the Galactic disk in the last $100 \mathrm{Myr}$, their results would favour an important dissolution rate of star clusters.

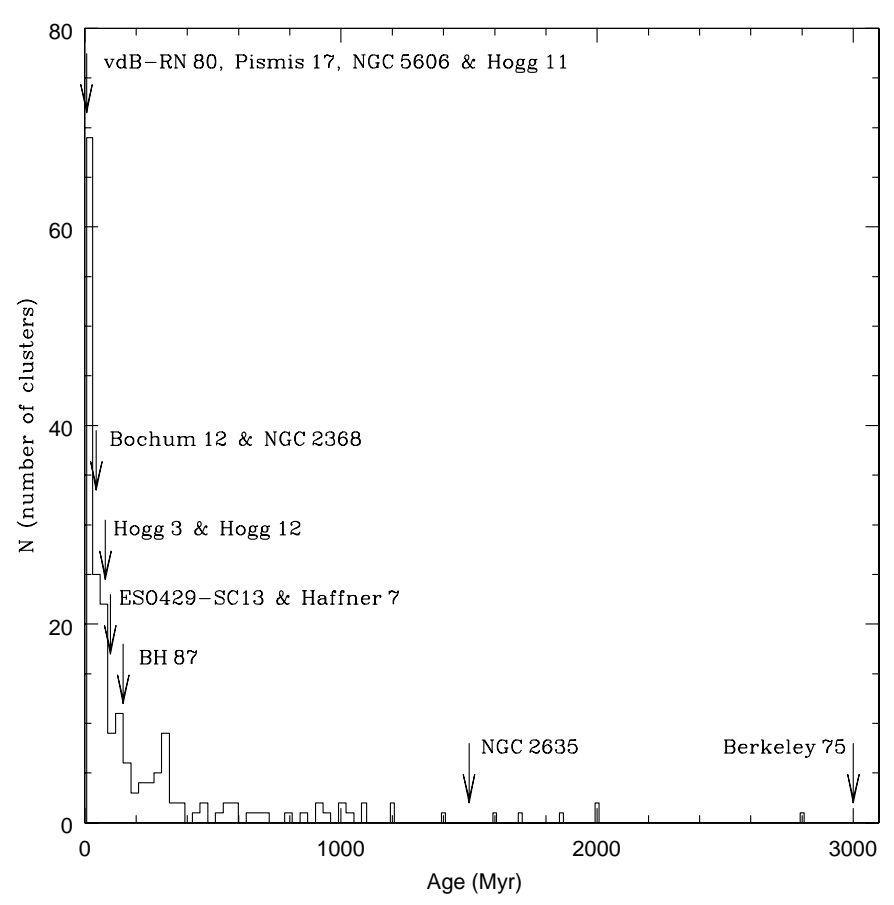

Fig. 8. Age histogram in the $90^{\circ}$ Galactic sector centered at $l=270^{\circ}$.

In the present study, the clusters are basically located within a $90^{\circ}$ sector centered at $l=270^{\circ}$. Figure 7 shows the reddening histogram in the above sector, which includes 198 clusters with $E(B-V)$ colour excesses taken from the WEBDA database. The reddening values for the current sample are indicated by arrows. The present clusters have a reddening value distribution typical of open clusters in this sector, i.e., not exceeding $E(B-V)=0.40$. An important fraction of literature clusters still exceed this value, but very few have a reddening value beyond $E(B-V)=1.0$. The age histogram (Fig. 8) shows that $85 \%$ of the present sample of open clusters correspond to the most frequent type, i.e., young blue open clusters. Two clusters (NGC 2635 and Berkeley 75) appear to be part of the relatively rare sample of intermediate-age open clusters. Likewise in Ahumada et al. (2000), the exploration of a sample of relatively faint optical open clusters, as compared to those already studied in detail in the same disk sector, indicates that the distributions of ages and reddening values are similar. The present results corroborate those in Ahumada et al. (2000), in the sense that the inclusion of fainter clusters maintains the age histogram distribution, which represents the formation/dissolution histories of open clusters, as seen from the solar locus in the Galaxy.

\section{Conclusions}

In the current paper we have considerably increased the sample of southern Galactic open clusters studied by means of integrated spectroscopy. Using template spectra and equivalent widths of the Balmer lines, we determined ages and foreground interstellar reddening 
values for 13 small size open clusters located in a $90^{\circ}$ sector centered at $l=270^{\circ}$. Nine of these concentrated objects have not been previously studied. The derived reddening values range from $E(B-V)=0.00$ in ESO 429-SC13 to $E(B-V)=0.38$ in vdB-RN 80. We found that Hogg 11, NGC 5606, vdB-RN 80 and Pismis 17 are very young clusters (4-8 Myr), while ESO 429SC13, Hogg 3, Hogg 12, Haffner 7, BH 87, NGC 2368 and Bochum 12 are moderately young (45-150 Myr). Two objects (Berkeley 75 and NGC 2635) exhibit spectral features typical of intermediate-age open clusters (1-2 Gyr). In spite of this, CMDs of these objects will be very useful to verify their tentative ages. The results derived here for Pismis 17, Bochum 12, Hogg 11 and NGC 5606, show good agreement with previous studies, except for the probable existence of internal reddening which cannot be inferred from the present templates. The current cluster sample improves the age resolution around solar metal content in the cluster spectral library for population synthesis. In a forthcoming study we will develop solar metallicity templates for different ages making use of the open cluster spectra in BA86a, Santos \& Bica (1993), Piatti et al. (1998a, 1998b, 1999, 2000a, 2000b), Ahumada et al. (2000) and the present data.

Acknowledgements. We are gratefully indebted to the CASLEO staff members and night assistants for their kind hospitality and support during the two observing runs. The authors acknowledge use of the CCD and data acquisition system supported under U.S. National Science Foundation grant AST-90-15827 to R. M. Rich. We gratefully acknowledge financial support from the Argentinian institutions CONICET, SECyT (Universidad Nacional de Córdoba) and AGENCIA CÓRDOBA CIENCIA and the Brazilian institutions CNPq and FINEP. We have used images from the Digitized Sky Survey (produced at the Space Telescope Science Institute under U.S. Government grant NAG W-2166) by means of the Canadian Astronomy Data Centre (CADC) interface.

\section{References}

Ahumada, A. V., Clariá, J. J., Bica, E., \& Piatti, A. E. 2000, A\&AS, 141, 79

Bica, E. 1988, A\&A, 195, 76

Bica, E., \& Alloin, D. 1986a, A\&A, 162, 21 (BA86a)

Bica, E., \& Alloin, D. 1986b, A\&AS, 66, 171 (BA86b)

Bica, E., \& Alloin, D. 1987, A\&A, 186, 49 (BA87)

Bica, E., Alloin, D., \& Santos, J. F. C. Jr. 1990, A\&A, 235, 103

Bica, E., Clariá, J. J., Bonatto, C., et al. 1995, A\&A, 303, 747

Bica, E., Clariá, J. J., Piatti, A. E., \& Bonatto, C. 1998, A\&AS, 131, 483

Bonatto, C., Bica, E., \& Alloin, D. 1995, A\&AS, 112, 71

Burki, G. 1975, A\&A, 43, 37

Clariá, J. J. 1976, AJ, 81, 155
Clariá, J. J., Mermilliod, J.-C., \& Piatti, A. E. 1999, A\&AS, 95, 429

Collinder, P. 1931, Medd. Lunds Astron. Observatorium 2

Feinstein, A. 1994, Rev. Mex. Astron. Astrof., 29, 141

Friel, E. D., \& Janes, K. A. 1993, A\&A, 267, 75

Frogel, J. A., Mould, J., \& Blanco, V. M. 1990, ApJ, 352, 96

García, B. 1994, ApJ, 436, 705

García, B., Clariá, J. J., \& Levato, H. 1988, Ap\&SS, 143, 317

Gum, C. S. 1955, Mem. RAS, 67, 155

Haffner, H. 1957, Z. Astrophys., 43, 89

Hogg, H. R. 1965a, Mem. Mount Stromlo, 17

Hogg, H. R. 1965b, PASP, 77, 440

Holmberg, E. B., Lauberts, A., Schuster, H.-E., \& West, R. M. 1978, A\&AS, 34, 285

Janes, K. A., \& Adler, D. 1982, ApJS, 49, 425

Janes, K. A., Tilley, C., \& Lyngå, G. 1988, AJ, 96, 771

Kholopov, P. N., Samus, N. N., Frolov, M. S., et al. 1998, The Combined Catalogue of Variable stars, 4th Edition, vol. II ("Nauka" Publishing House Moscow)

Lauberts, A. 1982, The ESO/Uppsala Survey of the ESO (B) Atlas, European Southern Observatory, Garching

Lyngå, G. 1987, Catalogue of Open Cluster Data, Strasbourg: Centre de Données Stellaires

Melotte, P. J. 1915, Mem. RAS, 60, 181

Mermilliod, J.-C. 1988, Bull. Inform. CDS, 35, 77

Mermilliod, J.-C. 1992, Bull. Inform. CDS, 40, 115

Meynet, G., Mermilliod, J.-C., \& Maeder, A. 1993, A\&AS, 98, 477

Minniti, D., Clariá, J. J., \& Gómez, M. 1989, Ap\&SS, 158, 9

Moffat, A. F. J., \& Vogt, N. 1973, A\&A, 10, 135

Moffat, A. F. J., \& Vogt, N. 1975, A\&A, 20, 125

Piatti, A. E., Clariá, J. J., \& Abadi, M. G. 1995, AJ, 110, 2813

Piatti, A. E., Bica, E., \& Clariá, J. J. 1998a, A\&AS, 127, 423

Piatti, A. E., Clariá, J. J., Bica, E., Geisler, D., \& Minniti, D. 1998b, AJ, 116, 801

Piatti, A. E., Clariá, J. J., \& Bica, 1999, MNRAS, 303, 65

Piatti, A. E., Bica, E., \& Clariá, J. J. 2000a, A\&A, 362, 959

Piatti, A. E., Clariá, J. J., \& Bica, E. 2000b, A\&A, 360, 529

Pismis, P. 1959, Bol. Tonantzintla y Tacubaya, 18, 37

Rodgers, A. W., Campbell, C. I., \& Whiteoak, J. B. 1960, MNRAS, 121, 103

Santos, J. F. C., Jr., \& Bica, E. 1993, MNRAS, 260, 915

Santos, J. F. C., Jr., Bica, E., Clariá, J. J., et al. 1995, MNRAS, 276,1155

Schmidt, A. 1988, SPEED User's Manual, Federal University of Santa María (Brazil)

Seaton, M. J. 1979, MNRAS, 187, 73p

Silva, D. R., \& Cornell, M. E. 1992, ApJS, 81, 865

Stephenson, C. B., \& Sanduleak, N. 1971, Publ. Warner \& Swasey Obs. 1, No. 1

Stone, R. P. S., \& Baldwin, J. A. 1983, MNRAS, 204, 347

van den Bergh, S. 1966, AJ, 71, 990

van den Bergh, S., \& Hagen, G. L. 1975, AJ, 80, 11

van den Bergh, S., \& Herbst, W. 1975, AJ, 80, 208

Vázquez, R. A., \& Feinstein, A. 1991, A\&AS, 87, 383

Vázquez, R. A., Baume, G., Feinstein, A., \& Prado, P. 1994, A\&AS, 106, 339

Vogt, N., \& Moffat, A. F. J. 1972, A\&A, 7, 133 